\title{
Ongoing evolution of KRAB zinc finger protein-coding genes in modern
}

\section{humans}

4 Christian W. Thorball ${ }^{1,2}$, Evarist Planet $^{1}$, Jonas de Tribolet-Hardy ${ }^{1}$, Alexandre Coudray ${ }^{1}$, Jacques

71 School of Life Sciences, École Polytechnique Fédérale de Lausanne, Lausanne, Switzerland.

82 Precision Medicine Unit, Lausanne University Hospital (CHUV) and University of Lausanne,

9 Lausanne, Switzerland.

\section{$11 *$ Corresponding author:}

12 Didier Trono

13 School of Life Sciences

14 École Polytechnique Fédérale de Lausanne

151015 Lausanne, Switzerland

16 E-mail: didier.trono@epfl.ch

20 Running title: Evolutionary and population dynamics of human KRAB zinc finger protein genes

Keywords: KRAB-ZFP, Genetic variation, Natural selection, Coding constraint, Paralogs 


\section{Abstract}

\section{Background}

Krüppel-associated box (KRAB) zinc finger proteins (KZFPs) constitute the largest and fastest

KZFPs serve as repressors of transposable element-embedded regulatory sequences (TEeRS) and that the evolutionary turnover of KZFP genes is mainly attributable to the changing transposable element

(TE) load of their hosts. However, how natural selection and genetic variation are shaping this process

31 is still poorly defined.

\section{Methods}

33 Genetic information was collected from nine primate species and 138,500 human genomes. Gene-

34 wide as well as functional amino acid position specific constraint was calculated across all human

35 KZFPs.

\section{Results}

37 We found that the most conserved KZFPs, some of which go back close to 400 million years, have been subjected to marked negative selection in the evolutionarily recent past and are very homogeneous within the human population. In contrast, younger, largely primate-restricted family members present evidence of less negative selection than the rest of genome and lower levels of coding constraint, particularly within the sequences encoding the functional sites of their zinc finger (ZF) arrays. We defined 33 sets of KZFP paralogs, which pairwise displayed a broad range of coding constraints differentials, with more recently emerged paralogs usually displaying a higher frequency of putatively deleterious mutations and missense variants within the functional sites of their ZF arrays than their source gene. Finally, we identified three KZFP genes more constrained in the genomes of individuals of African ancestry than in Europeans, with their modes of expression or DNA targets pointing to possible links between these inter-populational genetic differences and regional differences in the prevalence of some diseases. 


\section{Conclusions}

50 This work shows how the ongoing selection of KZFPs contributes to modern human genetic variation,

51 in particular through the constraint of putatively deleterious- and missense variants in functional

52 protein sites, and how ongoing interplays between environment and KZFP genes might be impacting

53 the biology of modern humans. 


\section{Background}

KRAB-containing zinc finger protein (KZFP) genes emerged approximately 420 million years ago (MYA) in the last common ancestor of coelacanth (Latimeria chalumnae), lungfish and tetrapods, and have expanded since then by gene and segment duplication to count more than 350 representatives in the human genome [1,2]. Their products are characterized by an $\mathrm{N}$-terminal KRAB domain and a C-terminal array of two to more than forty Cys2-His2 (C2H2) zinc fingers (ZFs) with sequence-specific DNA binding potential [3]. Close to $45 \%$ and $90 \%$ of human KZFPs are primateand mammalian-restricted, respectively. Most of these evolutionarily more recent KZFPs act as repressors of the transcriptional activity of transposable elements (TE)-embedded regulatory sequences (TEeRS) via the KRAB-mediated recruitment of KAP1 (KRAB-associated protein 1, also known as TRIM28), which serves as a scaffold for a heterochromatin-inducing complex $[1,4]$. The primary consequence of this effect is not to prevent further transposition since mutations inactivate most TEs targeted by KZFPs, but to facilitate the cooption of TEeRS into transcriptional networks [5]. In contrast, more conserved KZFP family members, which are often endowed with variant KRAB domains, do not recruit KAP1 but display other protein interactomes suggestive of functions such as maintenance of genome architecture or RNA processing [4]. Individual members of the KZFP family have been implicated in processes as diverse as adipogenesis, cell differentiation, genomic imprinting, metabolic control or meiotic recombination [6-14]. Since KZFPs from coelacanth, our most distant KZFP-encoding relative, bind the cognate KAP1, it is likely that KZFPs first emerged as TE-controlling repressors and from then on underwent continuous selection through turnover of their hosts' TE loads. While derivatives were occasionally produced that escaped this evolutionary flushing by development and exaptation of novel functions, the largest fraction of the KZFP gene pool of individual lineages is renewed over time, contributing to the species-specificity of regulatory networks. How much genetic drift occurs on a much shorter time scale, for instance since modern 
humans emerged and spread over the globe, is nevertheless unknown, which prompted the present study.

The recent availability of large human genome sequencing datasets such as the Genome Aggregation Database (gnomAD) [15] provides an opportunity to identify biologically essential human genes through their depletion in protein-altering variants, also known as coding constraint [16-20]. However, while gene-wide measures of constraint are useful for identifying haploinsufficient genes and for interpreting disease-associated variants, they do not capture biologically important, sitespecific constraints within protein domains. The DNA-binding specificity of KZFPs is dictated mainly by three amino acids in the alpha-helical region of each ZF, known as the zinc fingerprint [1]. Thus, missense variants in these positions alter the target preference and thereby the functional impact of a KZFP. At the same time, changes in the two cysteine or two histidine residues $(\mathrm{C} 2 \mathrm{H} 2)$ that stabilize each ZF through the recruitment of a zinc ion inactivate these structural domains altogether. By combining phylogenetics and large-scale population genetics, the present work examines recent changes of the KZFP gene pool in the human lineage. Its results strongly suggest that a large fraction of KZFP genes are subjected to ongoing selective pressures, and in return contribute to phenotypic differences between individuals.

\section{Methods}

\section{Map of the human KRAB Zinc Finger protein clusters}

KZFP pairs were detected and their age defined as described in [1]. In short, the human genome (hg19) was translated in 6 reading frames and scanned for zinc finger and KRAB domains using Hidden-Markov-Models (Pfam [21]: KRAB (PF01352) and zf-C2H2 (PF00096)). Hits for KRAB and Zinc Finger domains were combined based on proximity and strandness and then manually curated and integrated with existing gene or pseudogene annotations. Their age is based on sequencing similarity with orthologues in other species. For this analysis the position of each KZFP 
was reduced to a point located in the center between the KRAB and Zinc Finger domains. Alternative

104 chromosomes were ignored. The KZFP clusters were defined as having at least 3 KZFPs that are no more than $250 \mathrm{~kb}$ apart from the center of another member, consistent with [22]. The clusters are named after their chromosome and then numbered starting from the short arm of the chromosome.

107 The size of chromosomes and positions of centromeres were taken from UCSC genome browser annotation data [23] for hg19.

\section{Primate phylogeny and natural selection}

111 The time of divergence (i.e. branch lengths) between human, chimpanzee, gorilla, orangutan, 112 macaque, marmoset, tarsier, galago (a.k.a. bush baby) and mouse lemur was obtained from 10KTrees, 113 which uses Bayesian inference to estimate these [24].

114 Measures of natural selection in terms of $\mathrm{dN} / \mathrm{dS}$ across the nine primate species listed above was 115 obtained with PAML (v4.4) as previously described [25].

\section{Human genetic variation data}

118 Human genetic exome and whole genome sequencing data were obtained from The Genome 119 Aggregation Database (gnomAD) [16,26] (release-2.0.2) for 123,136 and 15,496 individuals, 120 respectively. The released genetic data was processed and filtered through several steps to guarantee 121 that only high-quality variants were included. First, all variants $+/-1 \mathrm{~kb}$ around the KZFP canonical 122 transcripts as defined by Ensembl (v75, hg19) were extracted and filtered for variant quality, thus 123 only retaining variants annotated as "PASS". Second, all indels were normalized and multiallelic 124 variants split using BCFTOOLS (v1.8) and reannotated with the Variant Effect Predictor [27] and 125 LOFTEE (v0.3beta). Third, all missense and loss of function (LoF) variants, defined as either 126 frameshift, stop-gain or splice variants, were extracted from both the exome and whole genome 127 datasets and either low confidence or flagged LoF variants were removed. The latter was primarily 
128 due to LoF variants found in the last $5 \%$ of the canonical transcript. Since genomic sequencing

129 methods can yield variable coverage of genetic regions, especially when it comes to exome 130 sequencing that is dependent on the capture of previously annotated protein-coding genes, we 131 excluded all canonical transcripts having an average per-base coverage $<20 \mathrm{x}$. Thus, bringing the total 132 number of included KZFPs to 361. Furthermore, exons with an average per-base coverage $<20 \mathrm{x}$ were 133 also removed, and the lengths of the coding sequences used later for normalizations were adjusted 134 accordingly. Finally, the filtered exome and genome datasets were combined, and the allele counts 135 and frequencies for all variants were recalculated, prior to the removal of all singletons (allele count $136=1)$ to hinder inflation of observed mutational events due to potential technical artifacts.

\section{Domain and site specifications}

139 The genomic positions of the $\mathrm{C} 2 \mathrm{H} 2$ zinc finger domains were obtained from the Ensembl database 140 (v75, hg19). For each KZFP, only the ones from the canonical transcripts (as defined by Ensembl) 141 were considered. The positions of the specific amino acids within these domains were 142 computationally annotated.

$143 \mathrm{Z}$ scores for the cysteine and histidine $(\mathrm{C} 2 \mathrm{H} 2)$ residues and the DNA fingerprint positions within the $144 \mathrm{ZF}$ domains were calculated with the number of missense variants normalized to the number of ZF 145 domains within the canonical transcript of each KZFP. For missense and LoF variants spanning either 146 the whole CDS or a full protein domain, the number of variants per gene, $x$, was normalized by the 147 length of the canonical coding sequence prior to $\mathrm{Z}$ score transformations.

$$
Z \text { score }=\frac{(x-\text { mean }(\text { variant count }))}{\text { sd(variant count })}
$$




\section{Zinc fingerprint evolutionary conservation score}

154 To distinguish the evolutionary conservation of each KZFP within paralog pairs and determine the source gene of the pair, each array of zinc finger DNA-binding amino acids were concatenated as a single sequence, separated by an artificially added character. To align, we used a customized version

adding a +100 score when the special character was matched by itself, and a -100 penalty when the then aligned globally with pairwise2.align from Biopython [28] using the modified matrix, an opening gap penalty of -20 , a gap extension penalty of 0 and no penalty for end gaps. For each alignment, the score was normalized by the alignment length and corrected for the participation of the special level), with weights of 0.5 to each terms.

$\mathrm{CAZF}_{\text {score }}=\mathrm{w}_{1} \cdot \frac{\mathrm{AL}_{\text {score }}}{\mathrm{AL}_{\text {perfect }}} \cdot \frac{\mathrm{Len}_{\text {seq }}}{\text { Len }_{\text {align }}}+\mathrm{w}_{2} \cdot \frac{\mathrm{ZF}_{\text {perfect }}}{\mathrm{ZF}_{\text {total }}} \cdot \frac{\text { Len }_{\text {seq }}}{\text { Len }_{\text {align }}}$

With ALscore being the Blosum80 alignment score of the alignment, ALperfect the score of the 169 alignment of the sequence against itself, Lenseq being the length of the sequence, Lenalign the length 170 of the alignment (= the longest of both sequence), ZFperfect the number of zinc finger that aligned 171 without mismatches and ZFtotal the total number of Zinc Finger contained in a given KZFP.

\section{ChIP-exo data}

174 ChIP-exo data in 293 T cells was obtained from [1] using overexpression of tagged KZFPs. Reads 175 were mapped to the human genome assembly hg19 using Bowtie2 short read aligner [29], using the 176 --very-sensitive-local. Peaks were called using MACS v1.4.2.1 [30] with defaults parameters with 
control as in [1] and with the parameter --keep-dup all. For the rest of the analyses, only peaks with

178 MACS score $>80$ were kept.

179 KZFP motifs were identified using RSAT and the peak-motifs function [31].

180 TE enrichment analyses from ChIP-exo data was performed using TEnrich

181 (https://github.com/alexdray86/TEnrich). TEnrich relies on a binomial exact test, which compares

182 the number of peaks on a TE family to what would be expected by random while taking the total

183 genomic span of each TE family into account. A peak is considered to be on a TE when the overlap 184 is above $50 \%$.

185 Gene ontology (GO) enrichment analyses from ChIP-exo data of ZNF114 and ZNF714 for biological 186 processes was performed using GREAT (v4.0.4) [32], using the default settings and significance 187 levels.

\section{Gene expression data and correlations}

190 Median gene expression counts across 40 tissues were obtained from GTEx (v7) [33]. The median 191 expression value of the various brain sections included in GTEx were combined into a single category named "Brain". KZFPs were considered expressed when transcripts per million (TPM) were above 0.3 TPM. Spearman correlations were used to assess the expression similarities of KZFP paralogs.

Statistics

196 All statistical analyses were performed using R (v3.6.0). Wilcoxon rank sum tests were used for all 197 paired analyses (e.g, primate vs. non-primate groups) and correlations were made using Spearman's rank correlation coefficient. 


\section{Results}

\section{Genomic distribution and recent evolution of human KZFP genes}

203 The KZFP gene family has been expanding primarily through gene or segment duplications, with minor contributions of translocation, retrotransposition, or recombination. As a consequence, KZFP genes are often grouped in clusters, with adjacent family members encoding proteins with significant

206 levels of amino acid similarity. We established a census of the chromosomal distribution of 466 207 juxtaposed $\mathrm{KRAB}$ and $\mathrm{C} 2 \mathrm{H} 2$ poly-zinc finger domains identified in the human genome, independent 208 of their presence or not in an annotated gene (Figure 1). We found $74 \%(n=344)$ of these domain 209 pairs to be located within one of 30 clusters, defined here as a group of at least three $\mathrm{KRAB} /$ poly-ZF 210 couples separated by less than $250 \mathrm{~kb}$. The majority $(\mathrm{n}=248)$ concentrated in 11 clusters on

211 chromosome 19. Using previously estimated evolutionary ages [1], we further determined that 212 isolated protein-coding KZFP genes tended to be older than their cluster-associated counterparts ( $p=$ 213 3.5e-6). This fits with the proposal that chromosome 19 is the main region of emergence of new 214 KZFP genes [34] and suggests that escaping the tumultuous environment of this chromosome 215 facilitated the fixation of older family members. An exception is the grouping of some of the most 216 ancient KZFPs, namely ZNF282, ZNF777, and ZNF783, in cluster 7.3 at the distal end of human 217 chromosome 7 long arm as previously noticed [35].

218 To complement this first analysis, we examined the recent evolution of KZFP genes in the primate 219 lineage as described previously [25]. For this, we determined their gene-wide ratio of non220 synonymous (missense) (dN) to synonymous (dS) substitutions ( $\mathrm{dN} / \mathrm{dS})$, based on genome sequence 221 data from human, chimpanzee, gorilla, orangutan, macaque, marmoset, tarsier, galago (a.k.a. bush 222 baby) and mouse lemur, that is, over $\sim 6$ to $\sim 74$ million years of divergence (Figure $2 \mathrm{~A}$ ). We found 223 KZFPs to have significantly higher $\mathrm{dN} / \mathrm{dS}$ values than genes coding for other proteins $(\mathrm{p}=1.46 \mathrm{e}-49)$, 224 including KRAB-less ZF proteins $(\mathrm{p}=3.04 \mathrm{e}-46)$ (Figure 2B), confirming previous observations $225[35,36]$. We also noted that the distribution of $\mathrm{dN} / \mathrm{dS}$ values was bimodal amongst KZFPs, with 
younger, primate-specific genes displaying higher scores than family members having emerged earlier in evolution $(\mathrm{p}=2.69 \mathrm{e}-21)$ (Figure $2 \mathrm{C}$ ) and the $\mathrm{dN} / \mathrm{dS}$ ratio of KZFP genes being anticorrelated with their estimated age $($ rho $=-0.61)($ Figure $2 D)$.

\section{Conservation-related gradient of human KZFP polymorphisms}

231 The coding constraint of a gene or fragment thereof reflects the strength of selective pressures imposed on its sequence, hence the relative functional importance of the corresponding protein or protein domain for a given species. Typically, highly constrained coding regions correspond to loci where mutations are either associated with disease or are completely absent because they cause sterility or embryonic lethality. To examine the coding constraints imposed on human KZFP genes, we examined genetic variation amongst 138,632 individuals (15,496 genomes and 123,136 exomes) cataloged in gnomAD v.2.0.2. After removing coding sequences with low coverage and dismissing singletons to reduce the impact of false positives resulting from sequencing or alignment errors, we extracted protein-altering variants (missense and predicted loss of function -LoF- by frameshift, gain

240 of stop codon or alteration of essential splice sites) within the canonical transcripts of all remaining 241 KZFPs $(n=361)$. For the estimation of gene-wide constraint, we normalized the number of variants 242 for the length of the canonical coding sequence and translated the result into a $\mathrm{z}$ score to standardize 243 values (Figure $3 \mathrm{AB}$ ). Accordingly, negative deviation from the mean was a sign of increased 244 purifying selection as a consequence of reduced frequency of protein-altering variants. However, we 245 did not correct for a theoretically expected number of mutations as frequently done in this type of 246 analysis because the unstable structure of the ZF array-coding region of KZFP genes renders this 247 parameter unpredictable [2]. Gene-wide, LoF and ZF domain-specific scores modestly correlated with previously measured $\mathrm{dN} / \mathrm{dS}$ ratios and with the age of the KZFPs (Figure 3C). Examining individual domains revealed that this association stemmed mainly from the ZF C2H2- and to a lesser extent, fingerprint-coding sequences. Of note, other codons of the ZF-coding regions displayed no 
significant constraint, confirming that essential positions in ZFs are limited to the structure-conferring cysteine and histidine residues and the target-defining fingerprint residues at positions $-1,+3$ and +6 of the ZF alpha helix [37].

Primate-restricted, younger KZFPs were significantly less constrained both in terms of LoF $(p=9.1 \mathrm{e}-$ 13) and missense variation $(p=1.7 e-6)$ than their older counterparts (Figure 3D), with the difference mainly residing in sequences coding the poly-ZF $(p=4.6 e-9)$ rather than the KRAB domain $(p=$ domains $\left(\mathrm{p}_{\mathrm{ZF} \text { other }}=0.002\right)$. Correlating with their age, isolated KZFP genes were more constrained at sequences encoding the ZF C2H2 residues $(p=0.001)$ and fingerprint-defining positions $(p=0.02)$ and displayed lower LoF scores $(\mathrm{p}=0.001)$ than their cluster-associated counterparts, consistent with their stabilization over longer evolutionary times (Figure 3E). However, coding constraints were also highly heterogeneous within most clusters, indicating that differential selective pressures are rapidly exerted on members of a same cluster (Figure S1).

No LoF variants were detected amongst all examined individuals for ZFP92, ZNF606, ZNF81, ZNF777, ZNF121, ZNF250, and ZNF597. The ZFP92, ZNF81, and ZNF777 genes were also devoid of any missense mutations in their $\mathrm{C} 2 \mathrm{H} 2$ - or fingerprint-coding positions, while some were detected in ZNF121, ZNF250, ZNF597, and ZNF606 albeit at extremely low allele frequencies. For a majority of other KZFPs $(\mathrm{n}=213)$, some heterozygous but no homozygous LoF variants were observed.

270 Nevertheless, a significant number $(\mathrm{n}=148)$ presented homozygous LoF variants in at least two 271 individuals, suggesting reduced constraint $(\mathrm{p}<2.22 \mathrm{e}-16)$ (Figure S2). On average, members of this subgroup had a younger estimated age than the rest of the KZFPs $(\mathrm{p}=2.9 \mathrm{e}-13)$. 
275 We examined coding constraints amongst 33 identified sets of KZFP paralogs, which we defined as products of gene duplication with $\geq 60 \%$ similarity between their zinc fingerprints [1]. Accordingly, for 28 of the 33 paralog pairs, all paralog pair members were located within the same chromosomal cluster. Significant differences were noted, especially at the $\mathrm{C} 2 \mathrm{H} 2$-coding positions, with some pairs 279 of paralogs displaying closely similar coding constraints (e.g., ZNF75A and ZNF75D) while others were markedly divergent (e.g., ZNF160 and ZNF665) (Figure 4A and Figure S3). The level of divergence was not related to the age of the paralog pairs, whether at $\mathrm{C} 2 \mathrm{H} 2$-coding- $(\mathrm{p}=0.21)$ or usually also the most conserved in evolution (Figure 4A). For instance, the $\sim 90$-million-year old (MYO) ZNF160 was markedly more constrained than its 29 MYO ZNF665 paralog (Figure S4), both at $\mathrm{C} 2 \mathrm{H} 2$-coding positions (Figure 4A) and across other features (Figure 4B). A closer examination of ZNF160 and ZNF665 zinc fingerprints revealed that some ZFs were completely constrained in both KZFPs, while others were more flexible (Figure 4C). ChIP-seq analyses confirmed that these proteins recognized closely related sequence motifs (Figure 4D) in overlapping 289 sets of genomic targets (Figure 4E), notably some LINE1 integrants (Figure S5). Furthermore, the two paralogs were noted to have roughly similar expression patterns of across 40 tissues according to the GTEx database (rho $=0.89$ ). More globally, we observed that more constrained KZFPs were generally expressed at higher levels and more ubiquitously than their more flexible counterparts 293 (Figure S6), in line with previous reports [16,38].

\section{Evidence of modern selective pressures on human KZFP genes}

To test whether KZFP constraints vary across human populations due to recent selective pressure, we extracted the variant information from the two largest subsets of individuals in the gnomAD database, i.e., 63,369 non-Finnish Europeans (NFE) and 12,020 African/African-Americans (AFR). We 299 calculated their population-specific constraint, adjusting for the difference in sample size to reduce 
the bias in variant discovery power. Correlation of missense constraints across all KZFP genes was strong $($ rho $=0.70$, Figure $\mathrm{S} 7 \mathrm{~A})$, but it was weaker at $\mathrm{C} 2 \mathrm{H} 2-($ rho $=0.52$, Figure $\mathrm{S} 7 \mathrm{~B})$ and zinc

found. We also observed comparable rates of KZFP LoF variants in the NFE and AFR populations

$304($ rho $=0.67$, Figure 5A), with 65 restricted to the NFE and 7 to the AFR subsets. While this difference might simply reflect a difference in sample sizes, some noteworthy outliers pointed to possible population-specific adaptation. We observed LoF variants in ZNF114, ZNF426, and ZNF714 in the 63,369 NFE and in a group of 12,897 Finnish (FIN) individuals, whereas none were found in the 12,020 AFR (Figure 5B). According to GTEx, ZNF714 and ZNF426 are expressed in most tissues, whereas ZNF114 expression is restricted to the thyroid (Figure 5C). The genomic targets of ZNF426 are yet to been identified. The results of ChIP-seq experiments performed in 293T cells overexpressing tagged forms of ZNF114 and ZNF714 [1] revealed rare peaks for the former, while the latter was found significantly enriched at a subset of HERVK integrants (Figure 5D). Gene ontology (GO) enrichment analyses yielded significant biological process terms only for ZNF114,

314 pointing to its involvement in chromatin silencing and wound healing (Figure S8).

\section{Discussion}

317 KZFPs are amongst the fastest evolving vertebrate genes. Amongst the 361 protein-coding family 318 members with good sequencing coverage in the human genome included in this study, $87 \%$ were 319 placental mammalian-restricted ( $\leq 105 \mathrm{MYO}$ ), and 33\% were primate-specific, down from $45 \%$ for 320 all putative primate-specific KZFP coding sequences as these comprise numerous annotated 321 pseudogenes [1]. The present work demonstrates that the evolutionary conservation of individual KZFPs correlates with the genetic constraint imposed on their coding sequences. Older KZFPs display lower degrees of genetic variation in the human population than their more recent counterparts, in particular at positions encoding amino acids predicted to dictate the DNA binding 
specificity of their products. Recent studies indicate that highly conserved KZFPs, some of which can be traced back to the emergence of tetrapods, only rarely bind to recognizable TE-derived sequences. They often associate with proteins that evoke essential functions such as RNA metabolism or chromosome architecture, and are usually devoid of paralogs [1,4]. Our observation that these genes are under severe coding constraints is therefore not surprising. In contrast, younger KZFPs almost universally target TEs, often have paralogs with zinc fingerprints suggestive of at least partly overlapping sets of genomic targets, and display a KAP1-centered protein interactome primarily consistent with transcriptional repression $[1,4]$. The greater genetic variation observed in the human population at positions determining the genomic targets of these recently emerged KZFPs may thus be explained by functional redundancy between closely related family members or by the absence of TEs forcing fixation of at least part of their ZF-coding sequences. Conversely, this also suggests that genetic variations in KZFP gene sequences, together with differences in the genomic distribution and sequence of their TE targets, participate in the phenotypic diversification of modern humans.

Differentials in coding constraint greatly varied within identified sets of unequivocal KZFP paralogs, being very narrow in some cases (e.g., ZNF75A and ZNF75D; ZFP30 and ZFP82) and quite broad in others (e.g., ZNF160 and ZNF665; ZNF181 and ZNF302). No single parameter could account for these differences. For instance, ZNF75A and ZNF75D both recognize the 3'end of KZFP genes, while ZFP30 and ZFP82 respectively bind LINEs and SINEs, that is, completely distinct sets of genomic targets. As well, ZNF679-ZNF716 and ZNF600-ZNF611 are two pairs of evolutionarily recent $(<20 \mathrm{MYO})$ paralogs, yet they present with coding constraint differentials that are negligible for the former and pronounced for the latter. Still, it is noteworthy that for paralogs of detectably distinct ages, the older one was found to be generally more constrained than its duplication product, recapitulating a trend noted for the KZFP family as a whole. This supports a general model whereby KZFP paralogs diversify the trans-regulatory space available for coping with new genomic targets resulting from genetic drift in the TE load of the host organism, while the corresponding parent genes 
keep controlling pre-existing cis-acting regulatory sequences, ensuring that physiology is maintained.

351 This mechanism also explains the high turnover in the pool of a lineage's KZFP genes, even if only 352 a fraction of newcomer genes is positively selected.

353 Our data indicate that evolutionary forces have also shaped the KZFP gene pool of modern humans 354 in the recent past. It is generally considered that Homo sapiens started durably settling out of Africa some 60,000 years ago and that the genetic diversity of these early migrants was reduced compared to individuals left behind owing to their comparatively smaller number and due to selection bottlenecks. Accordingly, for most genes, the number of observed polymorphisms is expected to be either equal or greater in individuals of African descent compared with Europeans or Asians, a trend verified for the vast majority of human KZFP genes. Thus, it is noteworthy that at least three family members, ZNF114, ZNF426, and ZNF714, all three several tens of million years old, exhibit the opposite pattern with LoF mutants absent in individuals of African descent but observed in a European subpopulation of similar size. The first, ZNF114, has not been functionally examined previously, but is expressed mainly in the thyroid, with a GO enrichment analysis pointing to its 364 involvement in wound healing. The second, ZNF714, has previously been associated with insulin resistance [39], a trait that exhibits substantial population-specific differences with Africans having significantly lower insulin sensitivity and higher insulin responses than Europeans [40]. The third, ZNF426, was previously found to bind to a Kaposi's sarcoma-associated herpesvirus (KSHV) transactivator and to reduce as a consequence the transcriptional activity of this virus, qualifying 369 ZNF426 as an innate antiviral restriction factor [41]. As KHSV is rare in Europe and the Americas 370 but endemic in Africa [42], it is tempting to speculate that the presence of this pathogen contributed 371 to the maintenance of functional ZNF426 alleles in the African population, and that this selective pressure was alleviated for descendants of humans having long left this continent. Further exploration of these various hypotheses will require the analyses of greater numbers of genomes from individuals 374 of non-European ancestry to rule out genetic drift as the source of these population variation 
differences, as well as functional experiments directly addressing the roles of ZNF114, ZNF426, ZNF714 and possibly other KZFPs displaying differential coding constraints in various human subgroups.

\section{Conclusions}

380 Overall, we provide evidence of the genome-wide selection and constraint of human KZFPs, even 381 within functionally important protein sites, and their role in shaping the current genetic variation 382 within this large family of genome-regulating proteins with implications for the biology of modern 383 humans.

\section{List of abbreviations}

386 AFR: African/African-Americans

C2H2: Cys2-His2

388 FIN: Finnish

389 GO: Gene ontology

390 KAP1: KRAB-associated protein 1, also known as TRIM28

391 KRAB: Krüppel-associated box

392 KSHV: Kaposi's sarcoma-associated herpesvirus

393 KZFP: Krüppel-associated box (KRAB) zinc finger protein (KZFP)

394 LoF: Loss-of-function

395 MYA: Million years ago

396 MYO: Million years old

397 NFE: Non-Finnish Europeans

398 TE: Transposable element

399 TEeRS: transposable element-embedded regulatory sequences 


\section{Declarations}

\section{Availability of data and materials}

405 The datasets supporting the conclusions of this article, the KZFP genomic map and calculated genetic 406 constraint scores are available in the Figshare repository with the accession 407 https://dx.doi.org/10.6084/m9.figshare.11663475 and https://dx.doi.org/10.6084/ 408 m9.figshare.11733333, respectively.

\section{Competing interests}

411 The authors declare that they have no competing interests

\section{$413 \quad$ Funding}

414 This study was supported by grants from the European Research Council (KRABnKAP, No. 268721; 415 Transpos-X, No. 694658) and the Swiss National Science Foundation (310030_152879 and 416 310030B_173337) to D.T.

\section{Authors' contributions}

419 C.W.T., J.F., P.T., and D.T. contributed to the conception and design of the study. C.W.T., E.P., 420 J.D.T., A.C., J.F., P.T., and D.T. contributed to the analysis and interpretation of data. C.W.T., J.D.T. 421 and D.T. contributed to the drafting the manuscript. All authors reviewed and approved the final 422 manuscript.

\section{Acknowledgements}

Not applicable 


\section{References}

1. Imbeault M, Helleboid P-Y, Trono D. KRAB zinc-finger proteins contribute to the evolution of gene regulatory networks. Nature. 2017;543:550-4.

2. Yang P, Wang Y, Macfarlan TS. The Role of KRAB-ZFPs in Transposable Element Repression and Mammalian Evolution. Trends Genet. 2017;33:871-81.

3. Urrutia R. KRAB-containing zinc-finger repressor proteins. Genome Biol. 2003;4:231.

4. Helleboid P-Y, Heusel M, Duc J, Piot C, Thorball CW, Coluccio A, et al. The interactome of KRAB zinc finger proteins reveals the evolutionary history of their functional diversification. EMBO J. 2019;0:e101220.

5. Pontis J, Planet E, Offner S, Turelli P, Duc J, Coudray A, et al. Hominoid-Specific Transposable Elements and KZFPs Facilitate Human Embryonic Genome Activation and Control Transcription in Naive Human ESCs. Cell Stem Cell. 2019;24:724-735.e5.

6. Chen W, Schwalie PC, Pankevich EV, Gubelmann C, Raghav SK, Dainese R, et al. ZFP30 promotes adipogenesis through the KAP1-mediated activation of a retrotransposon-derived Pparg2 enhancer. Nat Commun. 2019;10:1809.

7. Takahashi N, Coluccio A, Thorball CW, Planet E, Shi H, Offner S, et al. ZNF445 is a primary regulator of genomic imprinting. Genes Dev. 2019;33:49-54.

8. Ecco G, Imbeault M, Trono D. KRAB zinc finger proteins. Dev Camb Engl. 2017;144:2719-29.

9. Hayashi K, Matsui Y. Meisetz, a novel histone tri-methyltransferase, regulates meiosis-specific epigenesis. Cell Cycle Georget Tex. 2006;5:615-20.

10. Lupo A, Cesaro E, Montano G, Zurlo D, Izzo P, Costanzo P. KRAB-zinc finger proteins: a repressor family displaying multiple biological functions. Curr Genomics. 2013;14:268.

11. Quenneville S, Verde G, Corsinotti A, Kapopoulou A, Jakobsson J, Offner S, et al. In embryonic stem cells, ZFP57/KAP1 recognize a methylated hexanucleotide to affect chromatin and DNA methylation of imprinting control regions. Mol Cell. 2011;44:361-72.

12. Wagner S, Hess MA, Ormonde-Hanson P, Malandro J, Hu H, Chen M, et al. A broad role for the zinc finger protein ZNF202 in human lipid metabolism. J Biol Chem. 2000;275:15685-90.

13. Zeng Y, Wang W, Ma J, Wang X, Guo M, Li W. Knockdown of ZNF268, which is transcriptionally downregulated by GATA-1, promotes proliferation of K562 cells. PloS One. 2012; $7:$ e29518.

14. Yang P, Wang Y, Hoang D, Tinkham M, Patel A, Sun M-A, et al. A placental growth factor is silenced in mouse embryos by the zinc finger protein ZFP568. Science. 2017;356:757-9. constraint spectrum quantified from variation in 141,456 humans. Nature. 2020;581:434-43. 
16. Lek M, Karczewski KJ, Minikel EV, Samocha KE, Banks E, Fennell T, et al. Analysis of protein-coding genetic variation in 60,706 humans. Nature. 2016;536:285-91.

17. Bartha I, Rausell A, McLaren PJ, Mohammadi P, Tardaguila M, Chaturvedi N, et al. The Characteristics of Heterozygous Protein Truncating Variants in the Human Genome. PLOS Comput Biol. 2015;11:e1004647.

18. Havrilla JM, Pedersen BS, Layer RM, Quinlan AR. A map of constrained coding regions in the human genome. Nat Genet. 2019;51:88.

19. Gussow AB, Petrovski S, Wang Q, Allen AS, Goldstein DB. The intolerance to functional genetic variation of protein domains predicts the localization of pathogenic mutations within genes. Genome Biol. 2016;17:9.

20. Samocha KE, Kosmicki JA, Karczewski KJ, O’Donnell-Luria AH, Pierce-Hoffman E, MacArthur DG, et al. Regional missense constraint improves variant deleteriousness prediction. bioRxiv. 2017;148353.

21. El-Gebali S, Mistry J, Bateman A, Eddy SR, Luciani A, Potter SC, et al. The Pfam protein families database in 2019. Nucleic Acids Res. 2019;47:D427-32.

22. Huntley S, Baggott DM, Hamilton AT, Tran-Gyamfi M, Yang S, Kim J, et al. A comprehensive catalog of human KRAB-associated zinc finger genes: insights into the evolutionary history of a large family of transcriptional repressors. Genome Res. 2006;16:669-77.

23. Haeussler M, Zweig AS, Tyner C, Speir ML, Rosenbloom KR, Raney BJ, et al. The UCSC Genome Browser database: 2019 update. Nucleic Acids Res. 2019;47:D853-8.

24. Arnold C, Matthews LJ, Nunn CL. The 10kTrees website: A new online resource for primate phylogeny. Evol Anthropol Issues News Rev. 2010;19:114-8.

25. McLaren PJ, Gawanbacht A, Pyndiah N, Krapp C, Hotter D, Kluge SF, et al. Identification of potential HIV restriction factors by combining evolutionary genomic signatures with functional analyses. Retrovirology. 2015;12:41.

26. Karczewski KJ, Francioli LC, Tiao G, Cummings BB, Alföldi J, Wang Q, et al. Variation across 141,456 human exomes and genomes reveals the spectrum of loss-of-function intolerance across human protein-coding genes. bioRxiv. 2019;531210.

27. McLaren W, Gil L, Hunt SE, Riat HS, Ritchie GRS, Thormann A, et al. The Ensembl Variant Effect Predictor. Genome Biol. 2016;17:122.

28. Cock PJA, Antao T, Chang JT, Chapman BA, Cox CJ, Dalke A, et al. Biopython: freely available Python tools for computational molecular biology and bioinformatics. Bioinforma Oxf Engl. 2009;25:1422-3.

29. Langmead B, Salzberg SL. Fast gapped-read alignment with Bowtie 2. Nat Methods. 2012;9:357-9.

30. Zhang Y, Liu T, Meyer CA, Eeckhoute J, Johnson DS, Bernstein BE, et al. Model-based Analysis of ChIP-Seq (MACS). Genome Biol. 2008;9:R137. 
31. Thomas-Chollier M, Herrmann C, Defrance M, Sand O, Thieffry D, van Helden J. RSAT peakmotifs: motif analysis in full-size ChIP-seq datasets. Nucleic Acids Res. 2012;40:e31-e31.

32. McLean CY, Bristor D, Hiller M, Clarke SL, Schaar BT, Lowe CB, et al. GREAT improves functional interpretation of cis-regulatory regions. Nat Biotechnol. 2010;28:495-501.

33. GTEx Consortium. Genetic effects on gene expression across human tissues. Nature. 2017;550:204-13.

34. Lukic S, Nicolas JC, Levine AJ. The diversity of zinc-finger genes on human chromosome 19 provides an evolutionary mechanism for defense against inherited endogenous retroviruses. Cell Death Differ. 2013;21:381-387.

35. Liu H, Chang L-H, Sun Y, Lu X, Stubbs L. Deep Vertebrate Roots for Mammalian Zinc Finger Transcription Factor Subfamilies. Genome Biol Evol. 2014;6:510-25.

36. Emerson RO, Thomas JH. Adaptive Evolution in Zinc Finger Transcription Factors. PLOS Genet. 2009;5:e1000325.

37. Najafabadi HS, Garton M, Weirauch MT, Mnaimneh S, Yang A, Kim PM, et al. Non-basecontacting residues enable kaleidoscopic evolution of metazoan $\mathrm{C} 2 \mathrm{H} 2$ zinc finger DNA binding. Genome Biol. 2017;18:167.

38. Kapopoulou A, Mathew L, Wong A, Trono D, Jensen JD. The evolution of gene expression and binding specificity of the largest transcription factor family in primates. Evolution. 2015;n/a-n/a.

39. Crujeiras AB, Diaz-Lagares A, Moreno-Navarrete JM, Sandoval J, Hervas D, Gomez A, et al. Genome-wide DNA methylation pattern in visceral adipose tissue differentiates insulin-resistant from insulin-sensitive obese subjects. Transl Res. 2016;178:13-24.e5.

40. Kodama K, Tojjar D, Yamada S, Toda K, Patel CJ, Butte AJ. Ethnic Differences in the Relationship Between Insulin Sensitivity and Insulin Response. Diabetes Care. 2013;36:1789-96.

41. Yang Z, Wen H-J, Minhas V, Wood C. The zinc finger DNA-binding domain of K-RBP plays an important role in regulating Kaposi's sarcoma-associated herpesvirus RTA-mediated gene expression. Virology. 2009;391:221-31.

42. Minhas V, Wood C. Epidemiology and Transmission of Kaposi’s Sarcoma-Associated Herpesvirus. Viruses. 2014;6:4178-94. 


\section{Figure legends}

Figure 1. Genomic distribution of KZFP genes. Dots indicate relative chromosomal position of

KZFP genes (defined by juxtaposed KRAB- and ZF-coding domains), with color code indicative of age (grey for unassigned) and numbered clusters pointed to in black. Higher magnification of chromosome 19 is presented on top right.

Figure 2. Evolution of human KZFP genes in primate lineage. (A) Phylogenetic tree of primate species used to calculate natural selection of human genes, with branch length indicating approximate time of divergence in million years (MYA). (B) Distribution of PAML dN/dS values of natural selection for KZFPs (red), non-KRAB ZFPs (green) and all remaining genes in the genome (blue).

(C) dN/dS distribution of primate-specific KZFPs (blue) and older (red) KZFP genes. (D) Spearman correlation of the $\mathrm{dN} / \mathrm{dS}$ values and estimated age of KZFP genes.

Figure 3. Coding constraints of KZFP genes. (A) Schematic of genetic constraint Z score calculation. WGS/WES, whole genome/exome sequencing; LoF, loss of function variant; CDS, coding sequence. (B) Distribution of indicated Z scores; lower score indicates increased constraint compared to average of all KZFPs. Full KZFP, all variants within the canonical KZFP transcript;

545 KRAB domain, only variants in the KRAB domain; ZF domains, variants within the ZF domains; ZF 546 other, variants in non-functional positions within the ZF domains; FingerPrint, variants in the ZF 547 fingerprint positions; $\mathrm{C} 2 \mathrm{H} 2$, variants in the cysteine or histidine positions of the ZF domains; LoF, 548 loss of function variants (C) Spearman correlations between Z scores, level of natural selection 549 (PAML dN/dS) and estimated age of KZFPs. The colors and their intensity represent the direction 550 and strength of the correlations, with blue representing a positive- and read a negative correlation. 551 Only significant correlations after Bonferroni correction are shown. (D) Primate vs. non-primate 
KZFP constraint across indicated KZFP domains or residues. (E) Relative constraint of indicated regions for KZFPs inside vs. outside clusters.

Figure 4. Analysis of KZFP paralogs. (A) Distribution of $\mathrm{C} 2 \mathrm{H} 2$ constraint $\mathrm{Z}$ scores for indicated sets of KZFP paralogs, arranged from top to bottom according to difference within pairs. Each KZFP is colored according to their respective age, with the line separating them colored as the mean age of the pair. The paralog within each pair with the most conserved fingerprint across evolutionary time, as determined by their CAZF value, is marked by a triangle, while less conserved KZFPs are marked by a dot. The order of the y-axis labels corresponds to the order of the colored points on the graph. (B) Differential constraint Z scores for indicated domains of paralogs ZNF160 and ZNF665. (C) Zinc fingerprints of ZNF160 and ZNF665 with the scaled minor allele frequency (MAF) of identified missense variants indicated on the sides. Grey lines indicate identical zinc fingerprints (D) Consensus DNA binding motifs of ZNF160 and ZNF665. (E) Venn diagram of ChIP-exo peaks of ZNF160 and ZNF665 in 293T cells. (F) Expression levels in transcripts per million (TPM) of ZNF160 and ZNF665 across all tissues depicted in GTEx.

Figure 5. Population-specific constraint of human KZFPs. (A) Frequency of gnomAD-catalogued LoF variants in Africans (AFR) and Non-Finnish Europeans (NFE) per 10,000 individuals and kb of coding sequence. Grey line indicates the linear fit of the data. Common major KZFP outliers are annotated by name. (B) Same as in (A) crossing similarly sized groups of Finnish (FIN) and African individuals. (C) Expression levels in transcripts per million (TPM) for ZNF114, ZNF426, and ZNF714 across 40 tissues. (D) Enrichment of the genomic TE targets of ZNF114 and ZNF714 from ChIP-exo peaks in $293 \mathrm{~T}$ cells compared to the genome wide distribution of TEs. Stars indicate significant enrichments and grey bars non-TE genomic targets. 
bioRxiv preprint doi: https://doi.org/10.1101/2020.09.01.277178. this version posted September 1,2020. The copyright holder for this Figure 1 preprint (which was not certified by peer review) is the author/funder, who has granted bioRxiv a license to display the preprint in

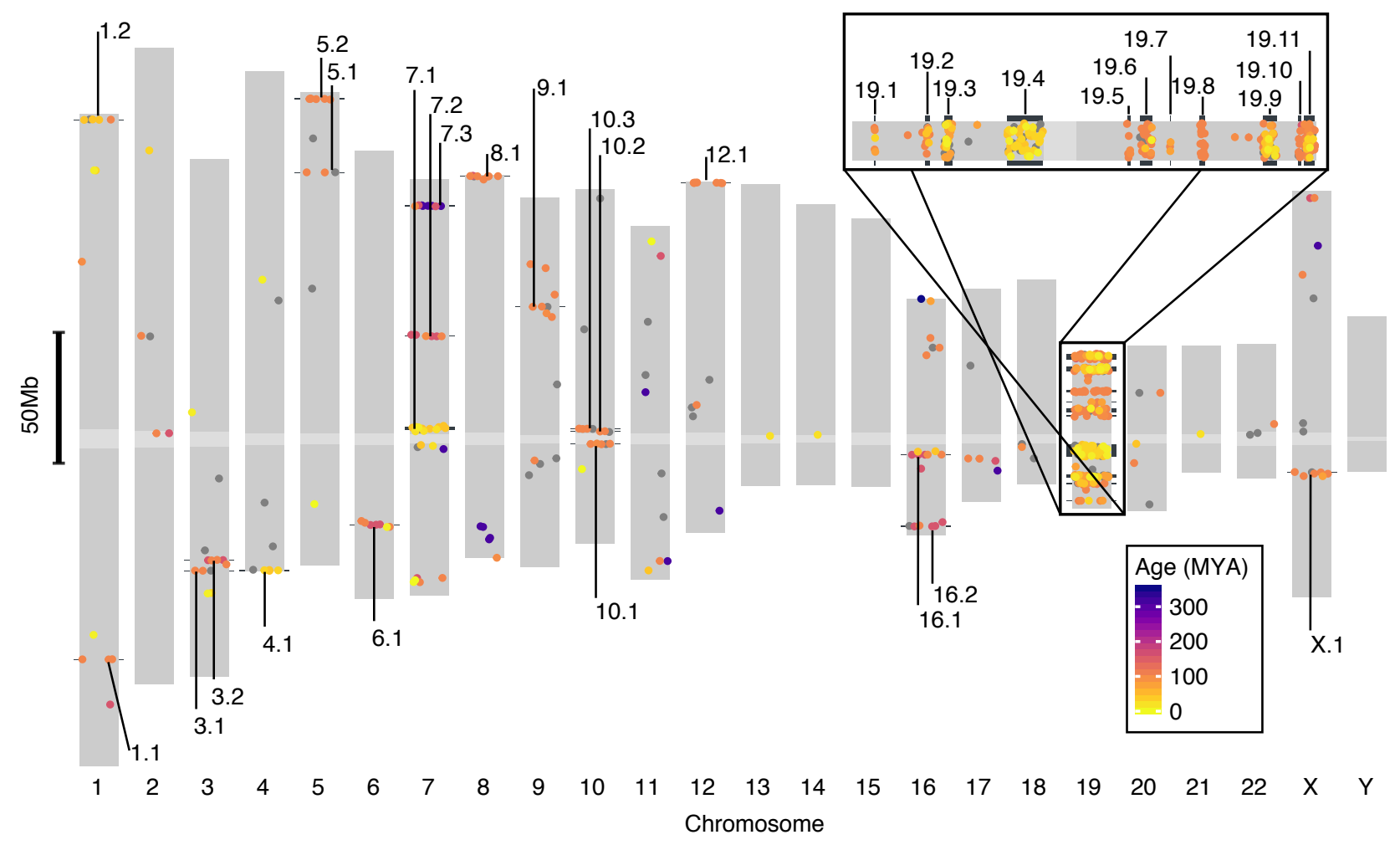


bioRxiv preprint doi: https://doi.org/10.1101/2020 09.01 277178; this version posted September 1, 2020. The copyright holder for this

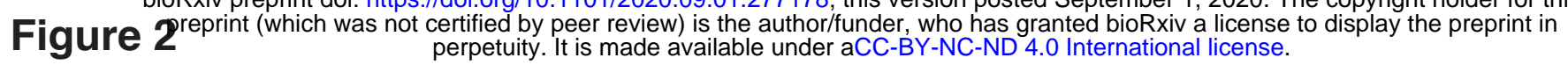

A

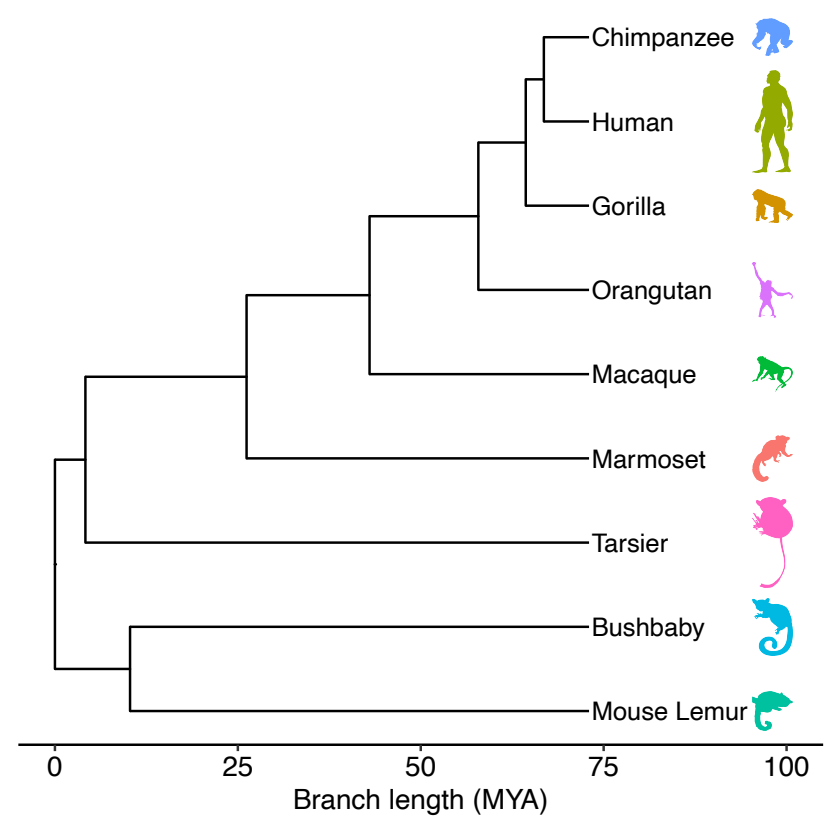

C

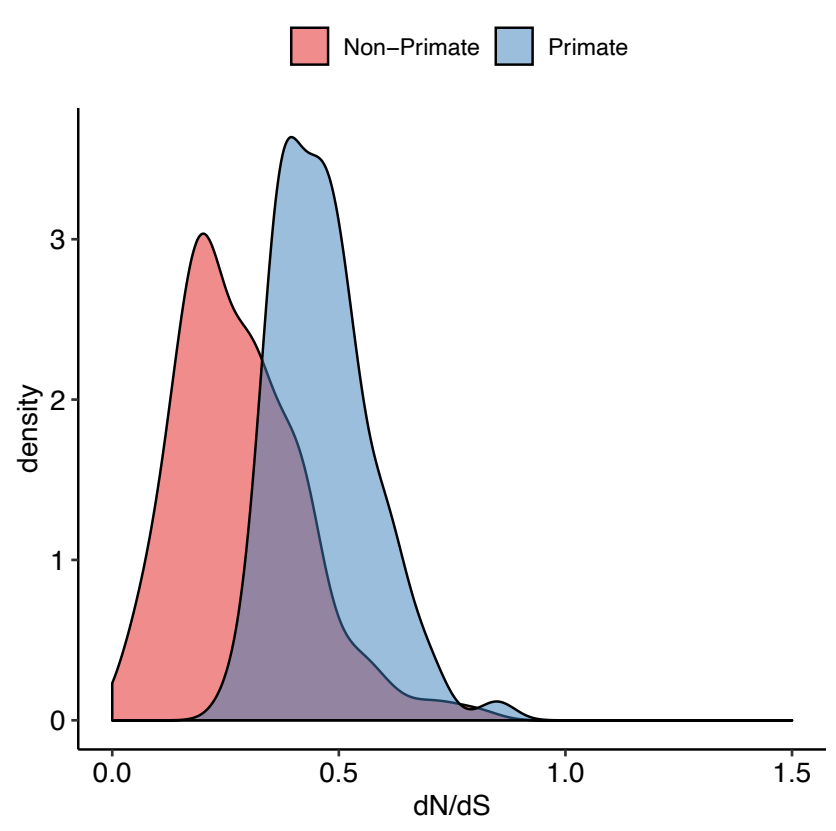

B

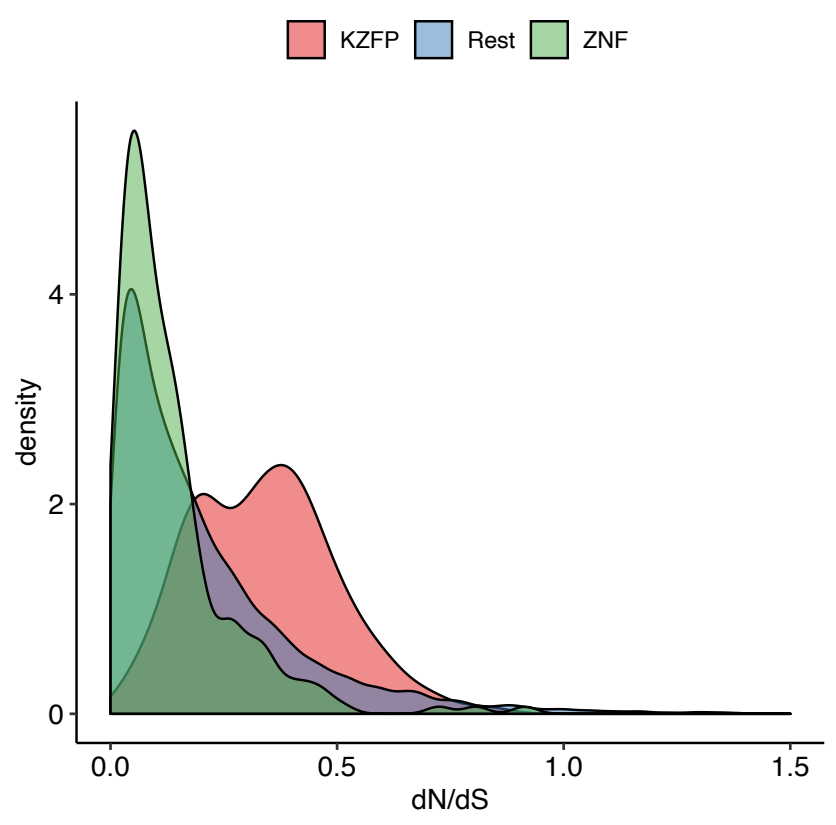

D

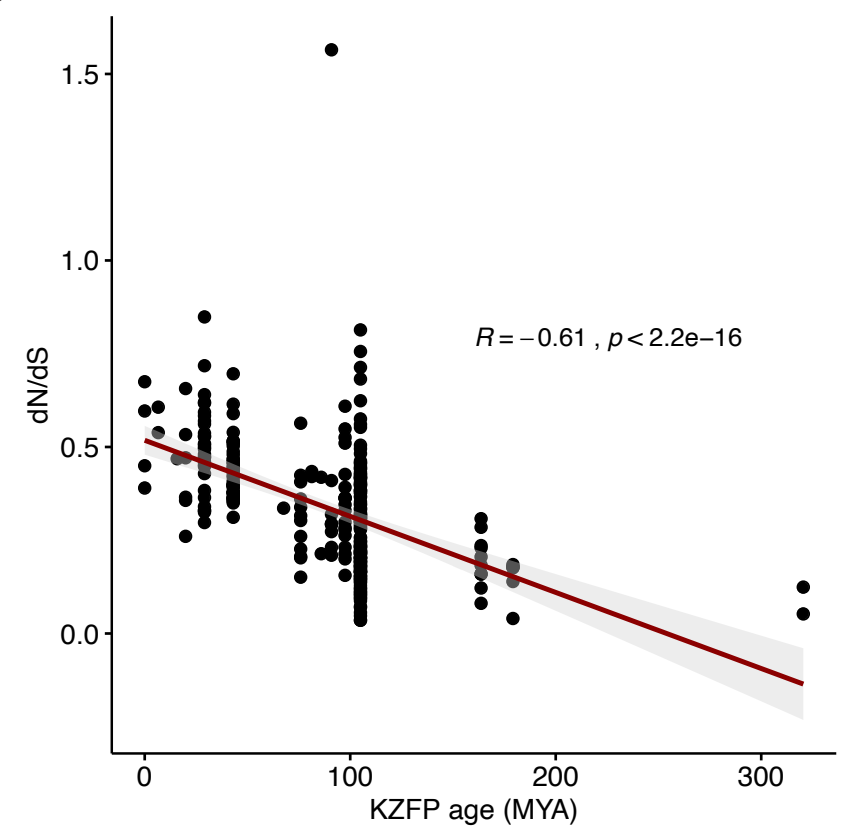


bioRxiv preprint doi: https://doi.org/10.1101/2020.09.01.277178; this version posted September 1, 2020. The copyright holder for this

Figure $3^{\text {preprint (which was not certified by peer review) is the author/funder, who has granted bioRxiv a license to display the preprint in }}$ perpetuity. It is made available under aCC-BY-NC-ND 4.0 International license.

A

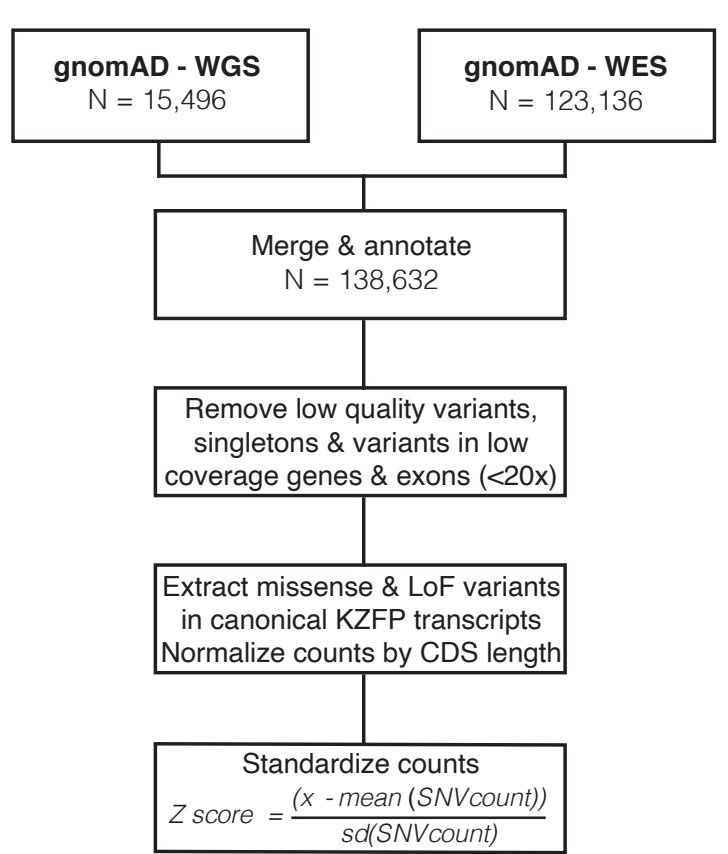

C

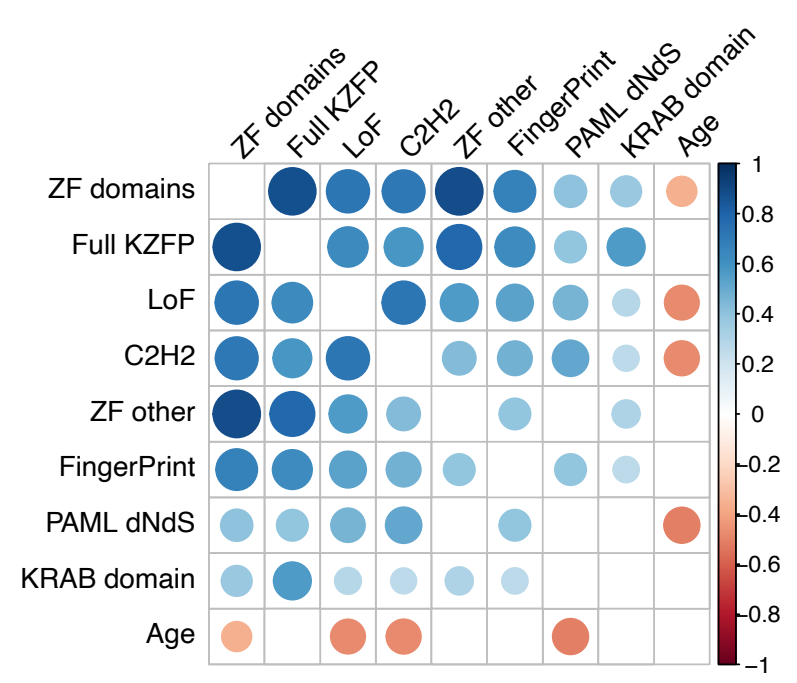

E

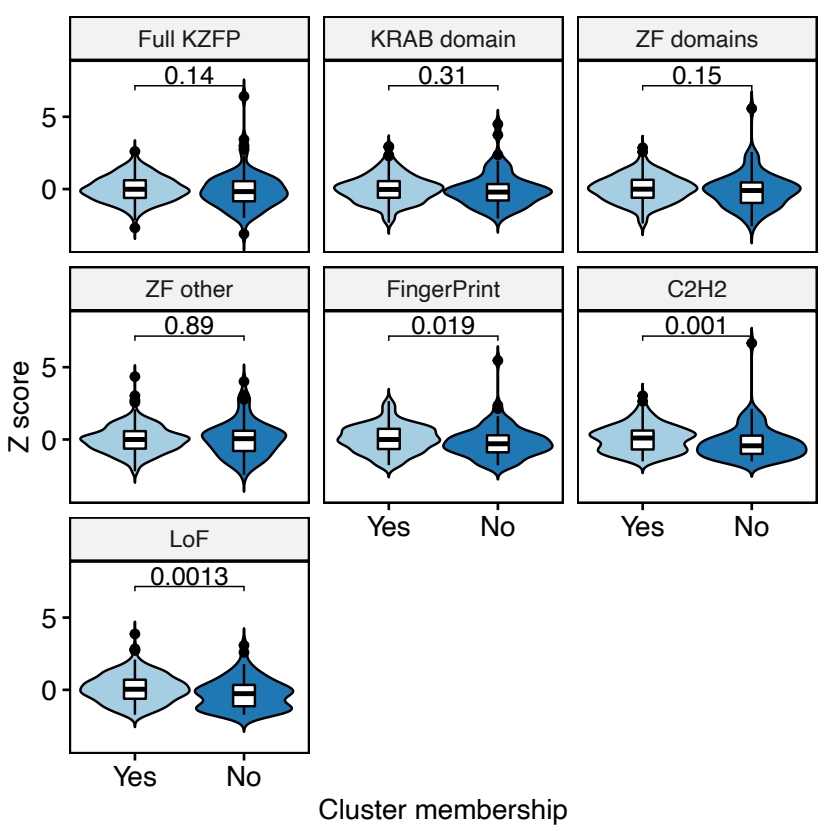

B

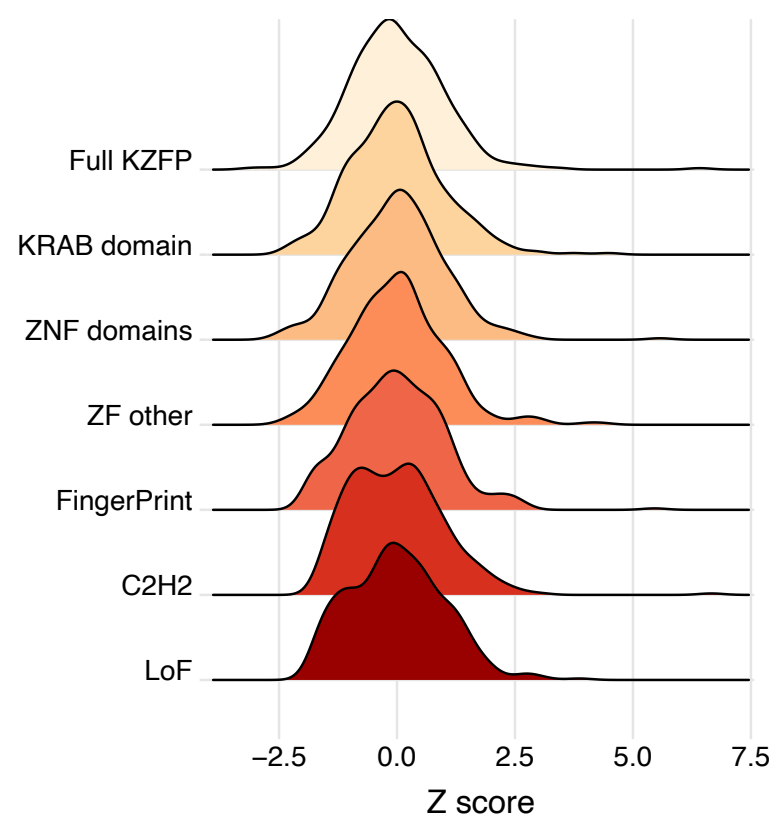

D

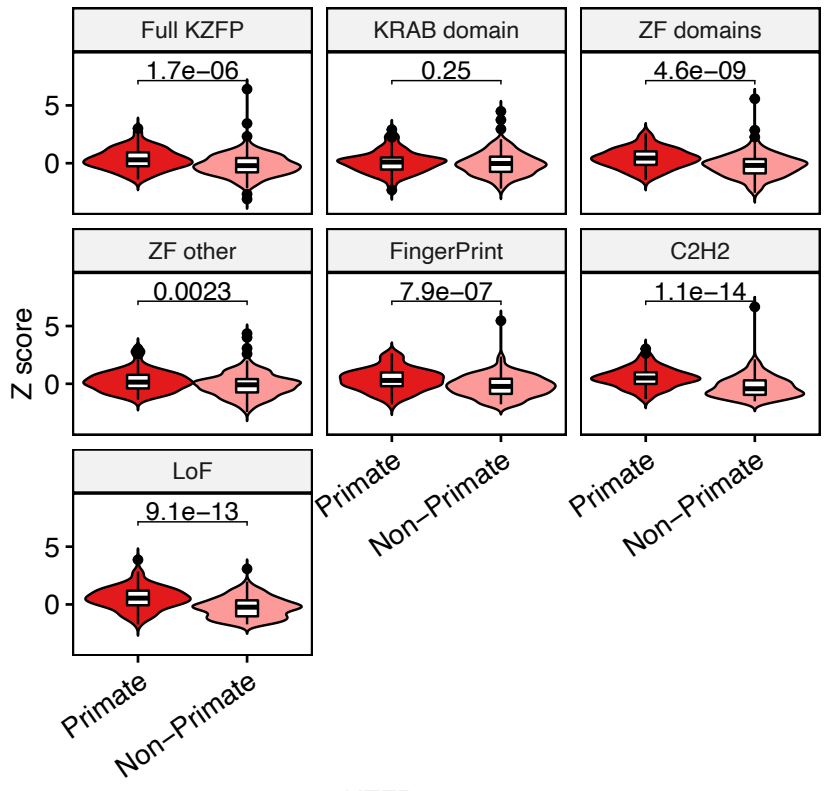

KZFP age group 
bioRxiv preprint doi: https://doi.org/10.1101/2020.09.01.277178; this version posted September 1, 2020. The copyright holder for this

preprint (which was not certified by peer review) is the author/funder, who has granted bioRxiv a license to display the preprint in

Figure 4 perpetuity. It is made available under aCC-BY-NC-ND 4.0 International license.

A

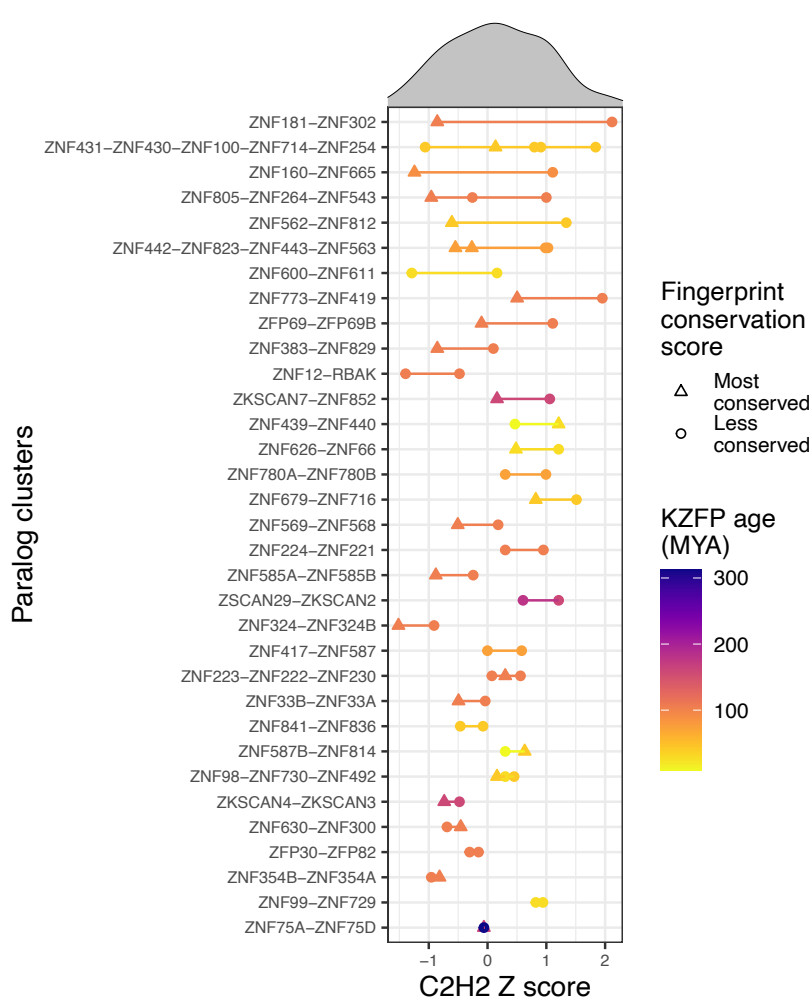

D
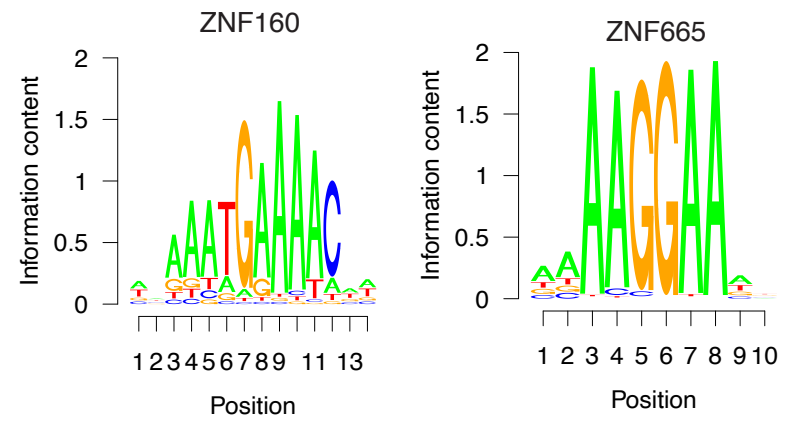

$\mathbf{F}$

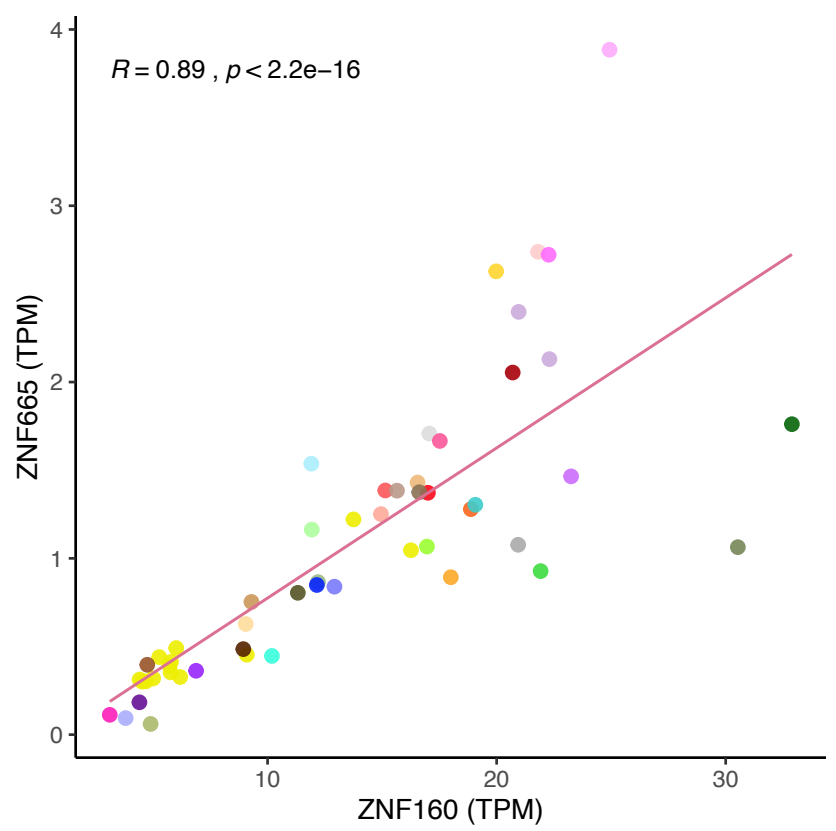

Cluster ZNF160-ZNF665

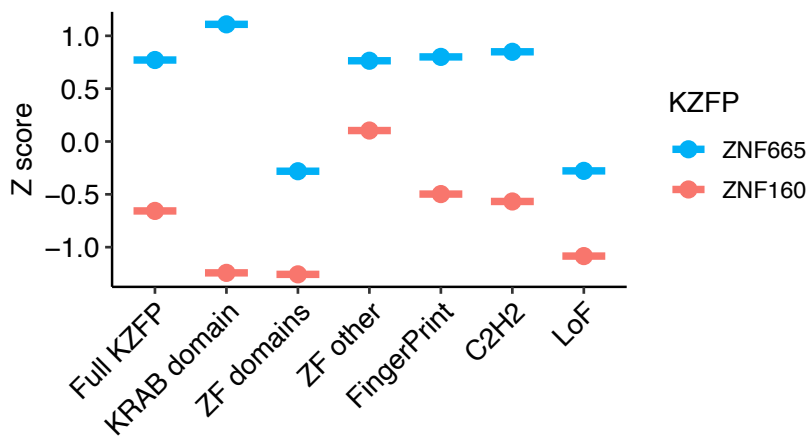

C

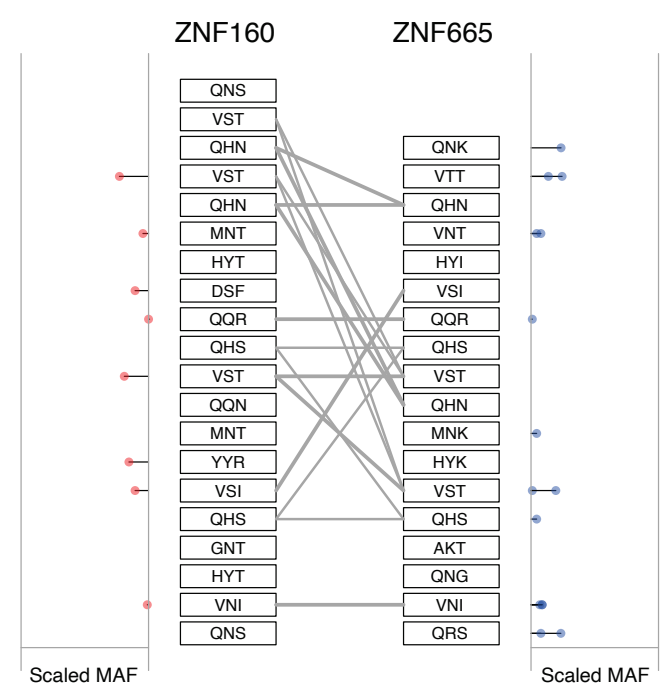

E

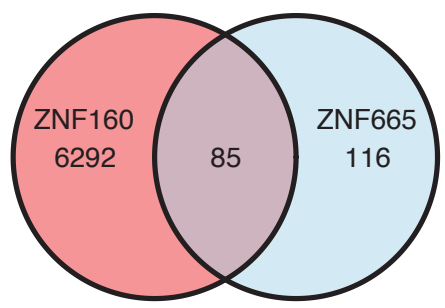

GTEx Tissues

- Adipose - Subcutaneous

- Adipose - Visceral (Omentum)

- Adrenal Gland

- Artery - Aorta

- Artery - Coronary

- Artery - Tibial

- Bladder

- Brain

- Breast - Mammary Tissue

- Cells - EBV transformed lymphocytes

- Cells - Transformed fibroblasts

- Cervix

- Colon - Sigmoid

Colon - Transverse

- Esophagus - Gastroesophageal Junction

- Esophagus - Mucosa

- Esophagus - Muscularis

Fallopian Tube

- Heart - Atrial Appendage

- Heart - Left Ventricle
Kidney - Cortex

- Liver

- Lung

Minor Salivary Gland

- Muscle - Skeletal

- Nerve - Tibial

- Ovary

- Pancreas

- Pituitary

- Prostate

- Skin - Not Sun Exposed (Suprapubic)

- Skin - Sun Exposed (Lower leg)

- Small.Intestine - Terminal lleum

Spleen

Stomach

- Testis

- Thyroid

- Uterus

- Vagina

- Whole Blood 
bioRxiv preprint doi: $\mathrm{https} / / /$ doi.org/10.1101/2020.09.01.277178; this version posted September 1, 2020. The copyright holder for this preprint (which was not certified by peer review) is the author/funder, who has granted bioRxiv a license to display the preprint in

\section{Figure 5}

A

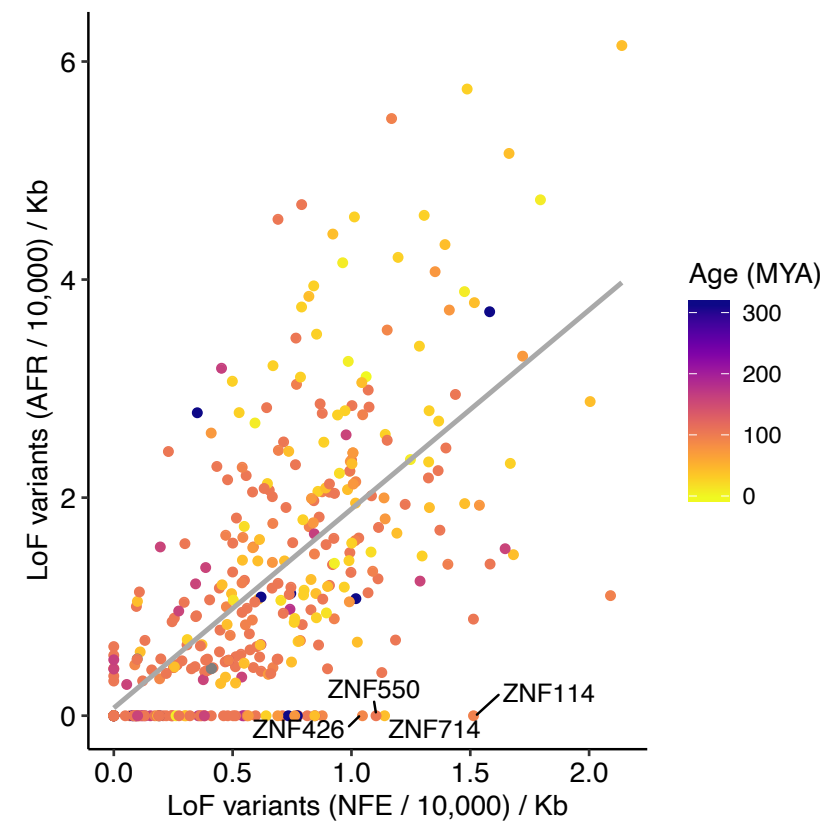

C

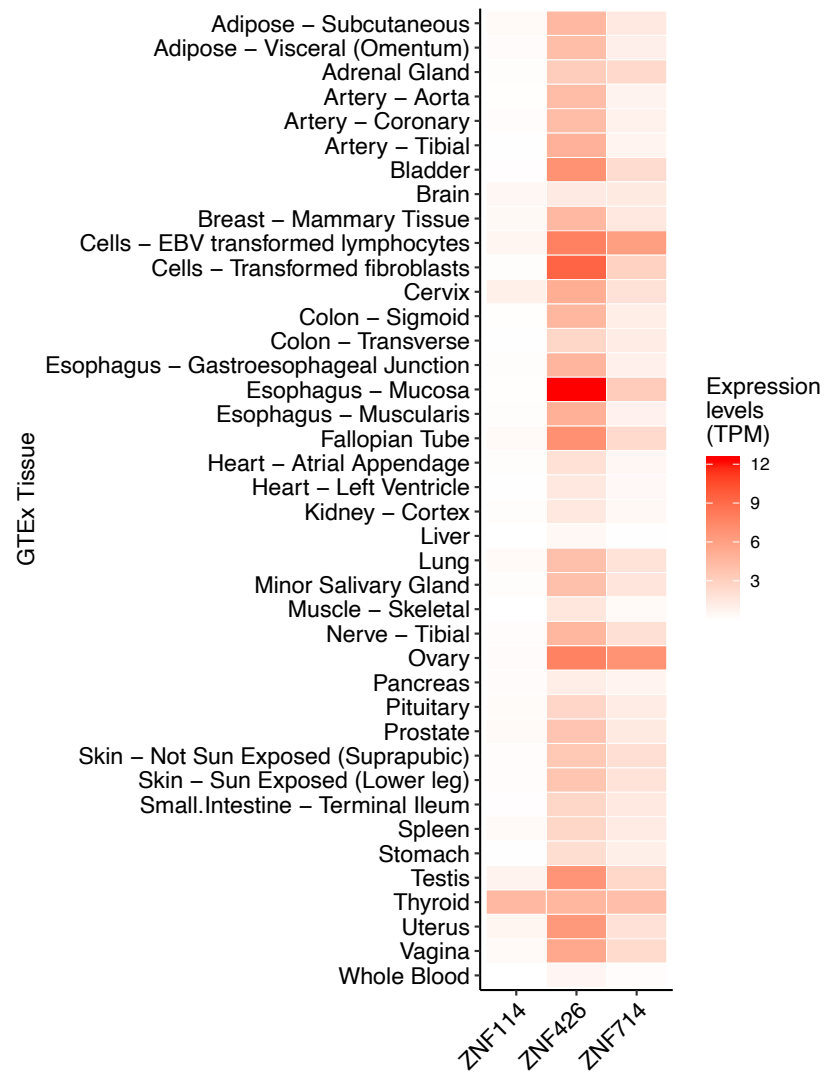

B

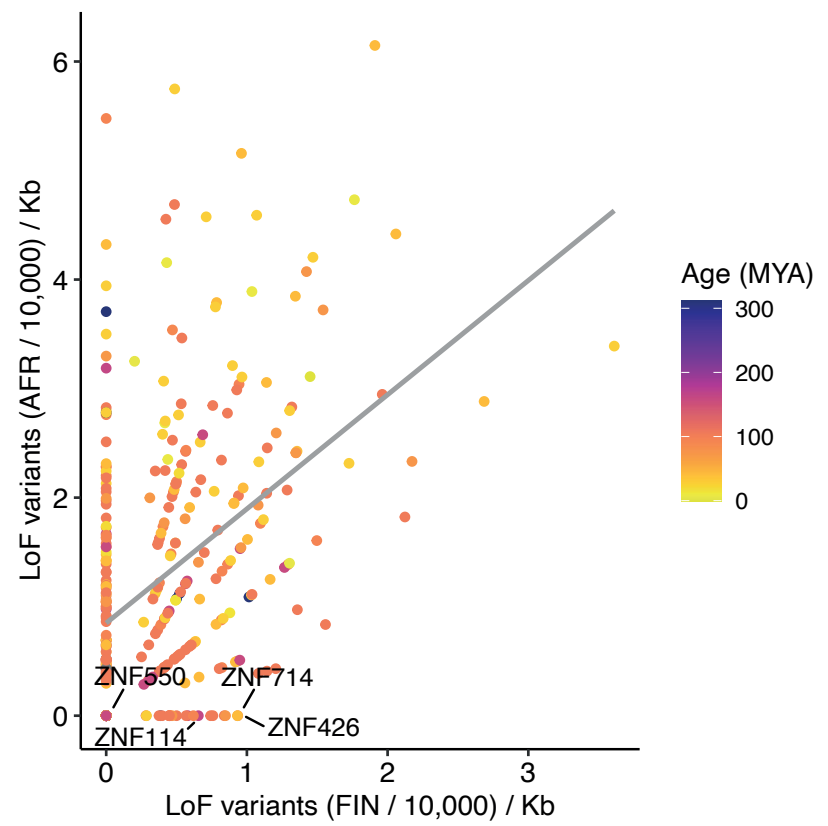

D

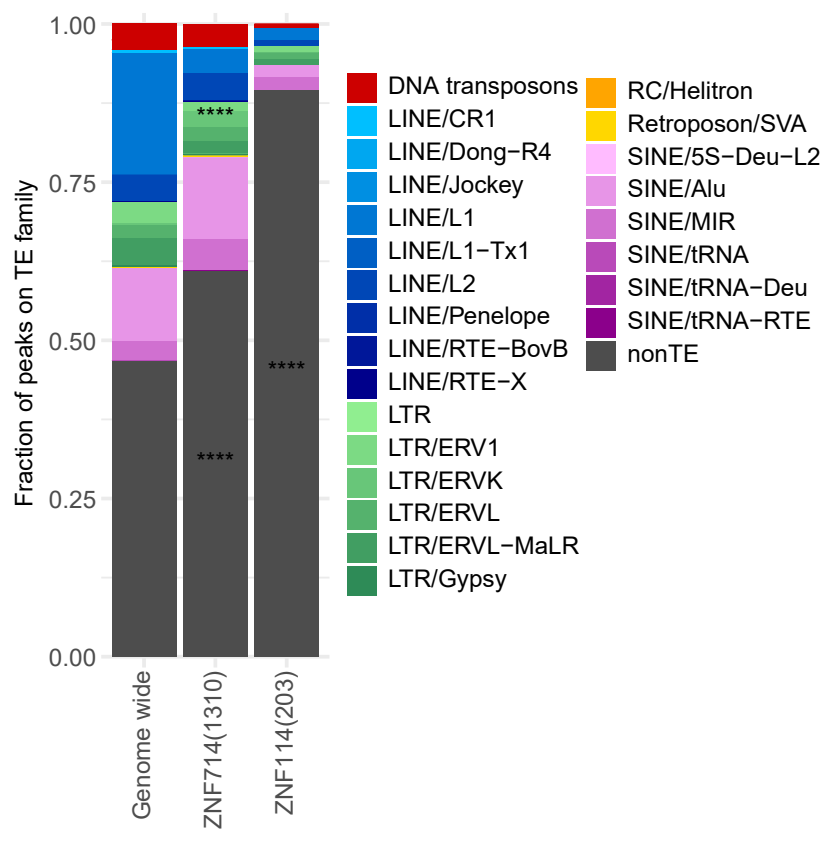

